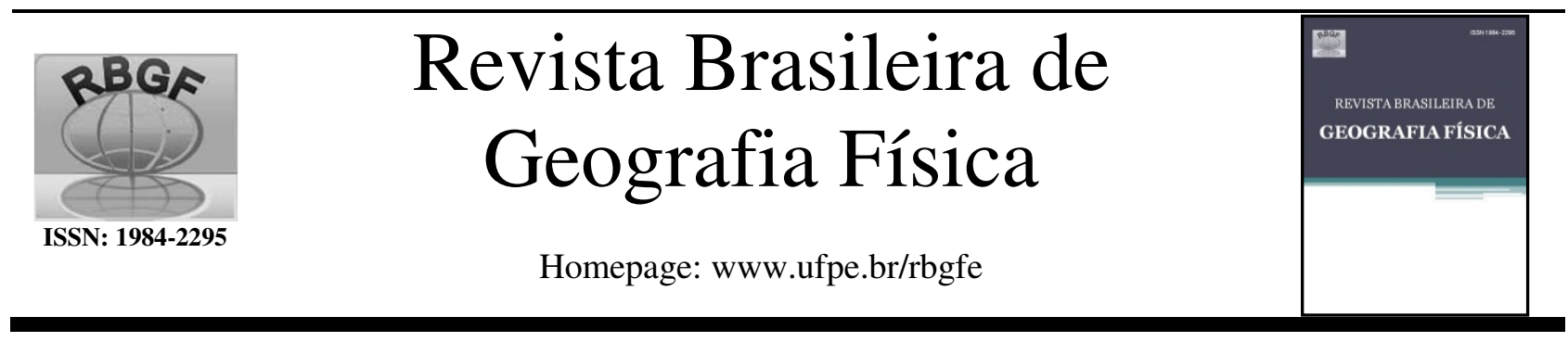

\title{
Análise dos Padrões Espaciais e Temporais da Precipitação no Estado do Maranhão-Brasil
}

Francisco das Chagas Araújo do Nascimento루 Fabiane Regina da Cunha Dantas Araújo²; Célia Campos Braga $^{3}$; Ewerton Vôlney da Silva Costa ${ }^{4}$

${ }^{1}$ Doutorando em Meteorologia, Universidade Federal de Campina Grande, UFCG-PB. Físico, Universidade Federal Rural do Semiárido, UFERSA-RN; ${ }^{2}$ Profa. Adjunta da Universidade Federal Rural do Semiárido, UFERSA-RN. E-mail: fabiane @ufersa.edu.br; ${ }^{3}$ Profa. Adjunta da UFCG-PB; ${ }^{4}$ Mestrando da Universidade Federal de Campina Grande, UFCG-PB.

Artigo submetido em 23/01/2015 e aceito em 24/03/2015

\section{R E S U M O}

Este trabalho objetivou verificar os padrões de precipitação pluvial no estado do Maranhão utilizando técnicas estatísticas da análise em multivariadas e relacioná-los com os principais fenômenos meteorológicos atuantes no estado. A área geográfica de estudo refere-se ao estado do Maranhão localizado no extremo Oeste do Nordeste do Brasil. Os dados de precipitação pluvial foram obtidos de 84 postos pluviométricos fornecidos pela Agência Nacional de Águas e Instituto Nacional de Meteorologia compreendendo o período de 1985 a 2014. Utilizou-se técnicas de Análise de Componentes Principais na definição dos padrões. Os resultados mostraram que nos meses de janeiro a abril as chuvas são mais intensas no norte do estado e no extremo com Tocantins, relacionadas à Zona de Convergência Intertropical, aos Vórtices Ciclônicos de Altos Níveis e influência Amazônica. Nos meses de maio a agosto a precipitação é predominante na parte noroeste influenciada pela Zona de Convergência Intertropical e Linha de Instabilidades e nos meses de setembro a dezembro ocorrem em maior quantidade no extremo Sul do estado influenciada pela Zona de Convergência do Atlântico Sul.

Palavras-chave: Análise de Componentes Principais, Regime Pluviométrico, Correlação.

\section{Analysis of the Spatial and Temporal Patterns of Rainfall in the State of Maranhão-Brazil}

\begin{abstract}
A B S T R A C T
This work aimed to verify the rainfall patterns in the state of Maranhão using statistical techniques the analysis in multivariate and relate them to the main meteorological phenomena operating in the state. The geographical area of study refers to the State of Maranhão located in the extreme west of Northeast Brazil. Rainfall data were obtained from 84 rain gauges provided by National Water Agency and National Institute of Meteorology covering the period of 1985-2014. We used techniques of Principal Component Analysis in the definition of standards. The results showed that in the months from January to April the rains are more intense in the northern state and in the extreme with Tocantins, related to the Intertropical Convergence Zone, the cyclonic vortices of high levels and Amazon influence. In the months from May to August the precipitation is predominant in the northwestern part influenced by Intertropical Convergence Zone and instabilities lines and in the months from September to December occur in larger quantities on extreme south of the state influenced by the South Atlantic Convergence Zone the cyclonic vortices of high levels.
\end{abstract}

Keywords: Principal Component Analysis, Rainfall Regime, Correlation.

\section{Introdução}

O Estado do Maranhão está localizado no extremo oeste do Nordeste Brasileiro (NEB) em

* E-mail para correspondência:
franciscofisica@ufersa.edu.br (Nascimento, F. da C.A. do). uma área dividida entre o complexo amazônico, onde o clima tende a ter características equatoriais; e outra situada na região semiárida. Essa localização atrelada a grande extensão territorial do estado são fatores condicionante do clima, e torna a distribuição da precipitação irregular. 
Vários sistemas de precipitação atuam sobre o Maranhão provocando grande variabilidade nas chuvas.

Dentre eles destacam-se a Zona de Convergência Intertropical (ZCIT), as Linhas de Instabilidades (LI), os Distúrbios Ondulatórios de Leste (DOL), a Zona de Convergência do Atlântico Sul (ZCAS), OS Sistema Frontais e os Vórtices Ciclônicos de Altos Níveis (VCAS).

Buscando uma boa compreensão da variabilidade de chuvas em uma determinada região, Lorenz (1956) introduziu o método da Análise em Componentes Principais (ACP), em estudos meteorológicos, as denominando de Funções Ortogonais Empíricas (FOE) para assim, destacar sua natureza não analítica (Braga, 2000). Estudos recentes mostram a importância dessa técnica em dados meteorológicos.

Santos et al. (2010) utilizaram ACP para encontrar relações entre padrões atmosféricos específicos, temporal e espacial da precipitação e formação de cheias no semiárido da bacia do rio São Francisco em 1985. Segundo os autores a atuação dos Vórtices Ciclônicos de Altos Níveis (VCAN) e da Zona de Convergência Intertropical (ZCIT) foram determinantes para $\mathrm{o}$ desenvolvimento de sistemas precipitantes intensos e ocorrência de inundações nos meses de janeiro a abril.

Amanajás e Braga (2012), através do método da ACP, analisaram os principais padrões climatológicos da precipitação na Amazônia Oriental e relacionaram com os principais mecanismos climáticos dos Oceanos Pacífico e Atlântico Tropical. Os resultados obtidos pela ACP mostraram a existência de três padrões pluviométricos bem definidos os quais, mostraram que as chuva são influenciadas pela atuação da ZCIT, LI e ZCAS.

Jones et al. (2014) utilizaram a ACP para apresentar uma representação das regiões que descrevem especificamente as características espaciais e temporais de chuvas extremas no Reino Unido. Os resultados confirmaram as tendências encontradas por outros autores, com o aumento do tempo de duração das chuvas nas últimas décadas no norte e oeste, e reduções no Sul do país.

Portanto, a técnica da análise multivariada tem apresentado bons resultados no tocante a um melhor entendimento dos padrões de variabilidade espaço-temporal da precipitação, que muitas vezes não ficam evidentes quando se trabalha apenas com seus valores médios ou sazonais (Stathis e Myronidis, 2009; Grimm, 2011). Nesse contexto, este trabalho objetivou verificar os padrões de precipitação pluvial no estado do Maranhão utilizando técnicas estatísticas da análise em multivariadas e relacioná-los com os principais fenômenos meteorológicos atuantes no estado.

\section{Material e Métodos}

A área geográfica de estudo refere-se ao Estado do Maranhão, localizado no extremo Oeste do Nordeste do Brasil entre $1^{\circ}$ e $10^{\circ}$ de Latitude Sul, e $41,5^{\circ}$ e $48,6^{\circ}$ de Longitude Oeste, com uma área de, aproximadamente, $331.983 \mathrm{~km}^{2}$. É limitado, ao Norte, pelo Oceano Atlântico, à Leste e Sudeste, pelo Estado do Piauí, ao Sul e Sudoeste, pelo Estado de Tocantins e, à Noroeste, pelo Estado do Pará. Os dados de precipitação pluvial foram obtidos de 84 postos pluviométricos distribuídos ao longo de todo estado (Figura 1) e fornecidos pela Agência Nacional de Águas (ANA) e Instituto Nacional de Meteorologia (INMET). Os dados dos postos compreendem o período de 1985 a 2014.

O cálculo da ACP é feito a partir da matriz de dados $\mathrm{X}$ ( $\mathrm{n}$ indivíduos $\mathrm{x} \mathrm{p}$ caracteres) com a qual se obtêm a matriz de variâncias e covariância $S$ através de:

$$
S=\frac{1}{n} X \cdot X^{t}
$$

onde: $X=x-\bar{x}$ e $X^{t}$ é a transposta de $X$. Sendo $\boldsymbol{R}$ uma matriz simétrica positiva de dimensão $(k \times$ $k$ ), ela é diagonalizável por uma matriz $\boldsymbol{A}$, de mudança de base, denominada de auto vetores. Logo:

$$
D=A^{-1} \cdot R \cdot A
$$

onde $\boldsymbol{D}$ é a matriz diagonal, cujos elementos $\lambda_{i}$ são os autovalores de a,ji. Pela ortogonalidade dos autovetores, a inversa de $\boldsymbol{A}\left(\boldsymbol{A}^{-1}\right)$ é igual a sua transposta $\left(A^{t}\right)$. Logo, as CPs $\mathrm{U}_{1}, \mathrm{U}_{2}, \ldots, \mathrm{U}_{\mathrm{p}}$ são obtidas por combinações lineares entre a transposta dos autovetores $\left(\boldsymbol{A}^{t}\right)$ e a matriz de observações $(\boldsymbol{X})$ padronizada. Cada linha de $\boldsymbol{U}$ corresponde a uma Componente Principal (CP) que forma as séries temporais associadas aos autovalores. Os valores de $X_{j}$ do n-ésimo local pode ser estimado por:

$$
\begin{gathered}
X=A \cdot U \\
X_{j}=a_{1 j} \cdot U_{1}+a_{2 j} \cdot U_{2}+\cdots a_{p j} \cdot U_{p}
\end{gathered}
$$




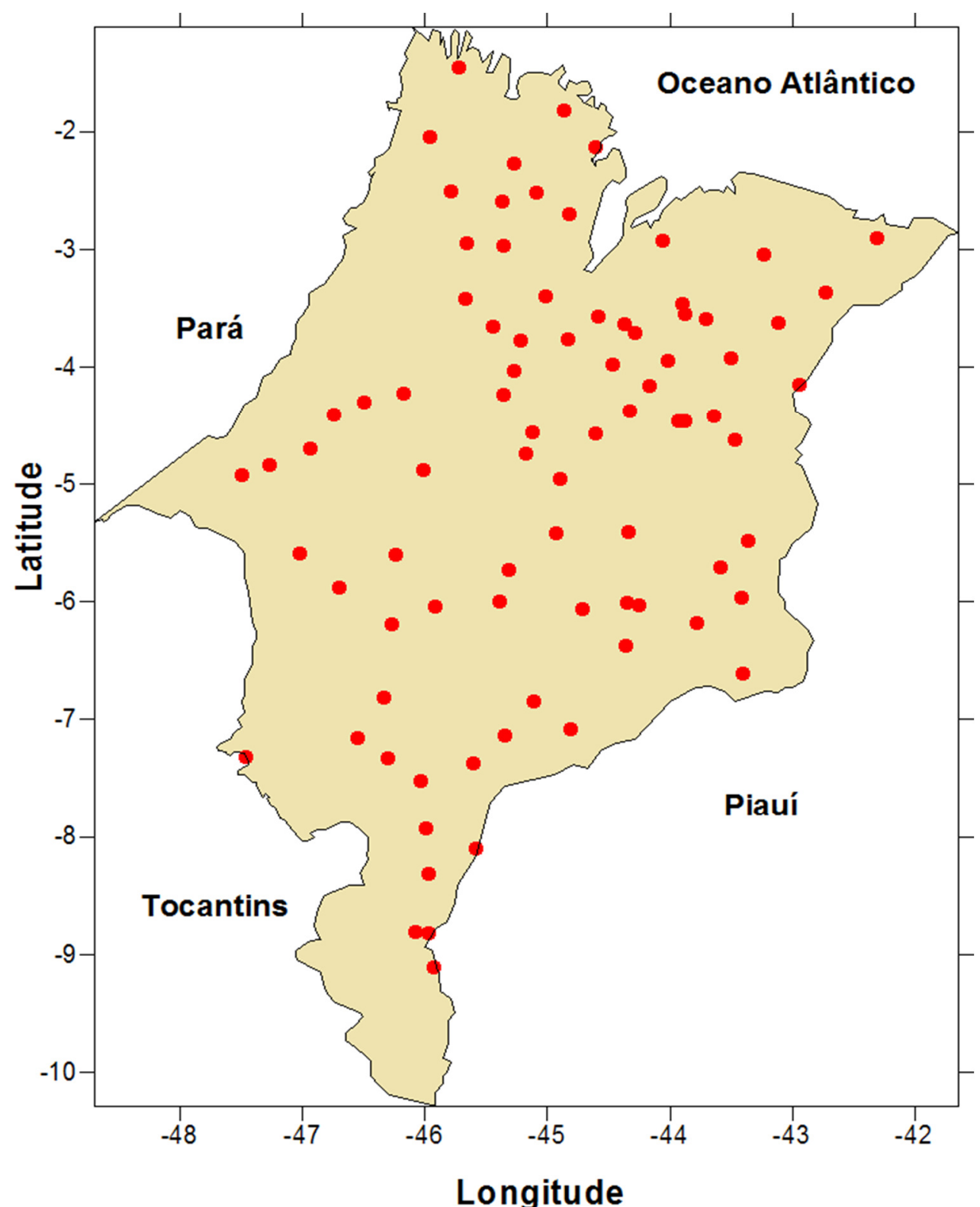

Figura 1. Distribuição espacial dos postos pluviométricos no Estado do Maranhão.

A solução desta equação é única. Esta solução considera a variação total presente no conjunto das variáveis iniciais, onde a $\mathrm{CP} 1$ explica o máximo possível da variância dos dados iniciais, enquanto a CP2 explica o máximo possível da variância ainda não explicada e assim por diante, até a última $\mathrm{CP}$ que contribui com a menor parcela de explicação da variância total dos dados iniciais. Cada CP tem uma porção da variância total dos dados de uma determinada variável e são ordenadas por ordem decrescente dos autovalores mais significativos de $a_{l}$ em $\boldsymbol{A}$, dada por:

$$
U_{k}=\sum_{j=1}^{p} a_{j k} \cdot X_{k}
$$

A partir da explicação da variância pela ordem decrescente dos autovalores se determina o melhor ajuste das CP. A soma dos valores próprios é a soma das variâncias centradas e normalizada;

$$
\sum_{i=1}^{n} \lambda_{i}=\sum_{i=1}^{n} \operatorname{var}\left(X_{i}\right)
$$

Desse modo, a porcentagem da variância dos autovalores em ordem decrescente é dada por:

$$
\operatorname{var}(X)=\frac{\sum_{i=1}^{q} \lambda_{i}}{\sum_{j=1}^{p} \lambda_{j}}
$$

A correlação entre a i-ésima variável original e a i-ésima componente principal é obtida por:

$$
\operatorname{coor}\left(X_{i} \cdot U_{j}\right)=a_{i j} \cdot \sqrt{\lambda_{i}}
$$

onde; $a_{i j}$ é o j-ésimo elemento do i=ésimo autovetor e $\lambda_{i}$ o i-ésimo autovalor. 
Por fim, foi feita a classificação dos grupos utilizando o método de Ward (1963) o qual é hierárquico e utiliza a distância euclidiana para medir a similaridade ou dissimilaridade entre os indivíduos. Este método propõe que em qualquer fase da análise a perda de informação que resulta do agrupamento de elementos entre grupos seja medida pela Soma dos Quadrados dos Desvios (SQD) de cada ponto à média dos elementos do grupo à qual pertence (Everitt, 1993).

\section{Resultados}

São apresentados e discutidos os resultados dos principais padrões espaciais e temporais da precipitação expressos pelos fatores comuns mais significativos obtidos a partir da Análise Fatorial em Componentes Principais e as regiões homogêneas determinadas a partir dos fatores comum espacial e temporal da precipitação retidos segundo critério de Kaiser. Os três primeiros fatores comuns temporais rotacionados explicaram $92,8 \%$ da variância total da precipitação mensal.

O primeiro fator que explica $34,6 \%$ da variância total apresenta correlações superiores a 0,8 nos meses de setembro a dezembro (Figura 2). O padrão espacial associado a este fator apresenta contribuições superiores a 1,0 no sul e extremo sudoeste; em um pequeno núcleo próximo a latitude $-3^{\circ} \mathrm{S}$ e no extremo noroeste na divisa com o estado do Pará as contribuições são superiores a 0,5 e nas demais regiões os valores são menores que $-0,5$ (Figura 3 ).

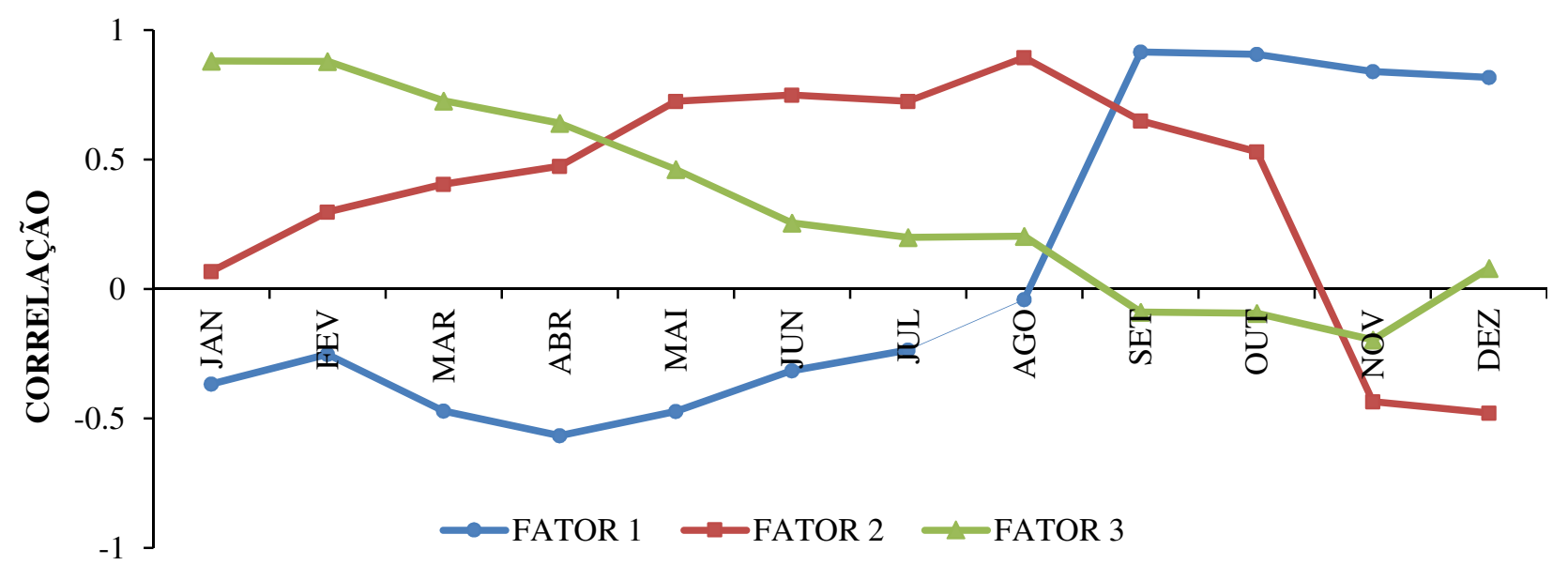

Figura 2. Correlações dos três primeiros fatores comuns temporais que explicam $92,8 \%$ da variância total dos dados da precipitação no Estado do Maranhão.

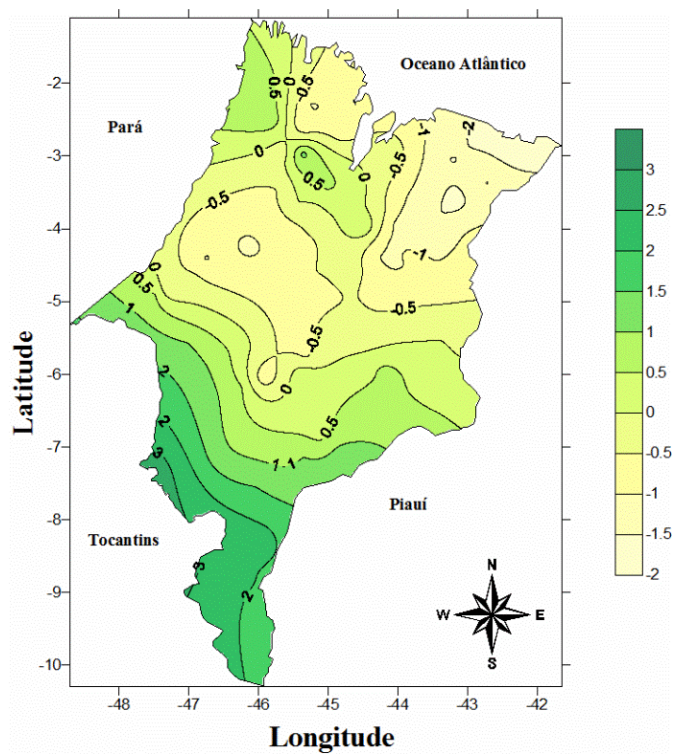

Figura 3. Padrão espacial do primeiro fator comum da precipitação (escores), no Estado do Maranhão para o período de 1985 a 2014.
O regime climatológico nos meses de setembro a dezembro na região Sul do Maranhão sofre influência da ZCAS, pois esta provoca uma banda de nebulosidade e chuva que se estende desde o noroeste da Amazônia até o Atlântico Sul Central no sentido noroeste-sudeste passando por sobre a parte sul e sudoeste do Estado provocando chuvas elevadas. Em associação com a ZCAS, existe na região outra contribuição importante, a Zona de Convergência de Umidade (ZCOU), que tem características parecidas com a ZCAS, atuando em dias diferentes. Outro fator contribuinte para a formação de chuvas na região são os Sistemas Frontais.

O segundo fator comum, que explica $33,9 \%$ da variância dos dados, tem altas correlações acima de 0,7 nos meses de maio a agosto (Figura 2). A configuração espacial deste fator têm contribuições elevadas acima de 2,0 no extremo noroeste. No restante do estado são menores que 0,5 , atingindo valores inferiores a $-0,5$ no extremo sul (Figura 4). 


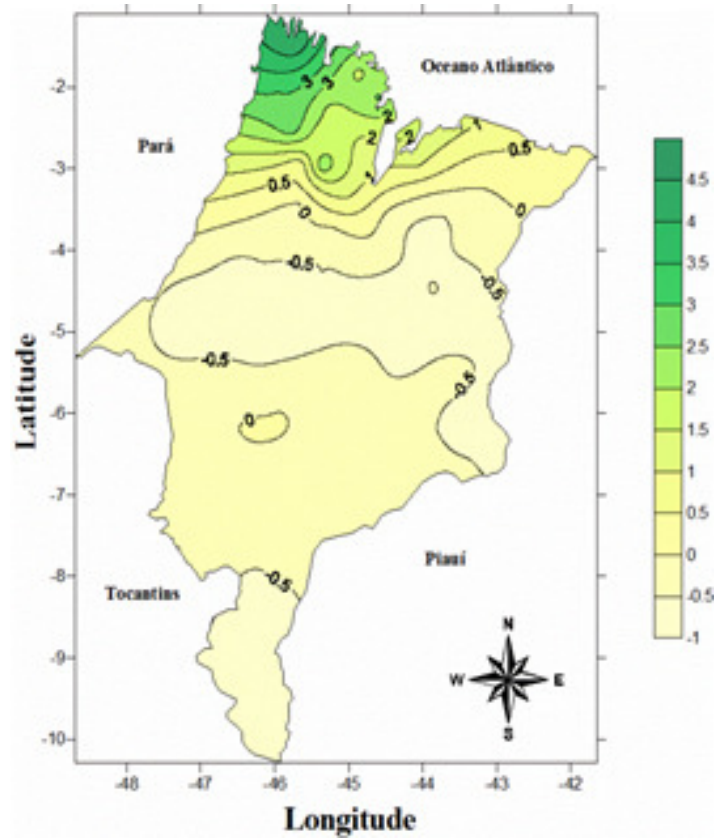

Figura 4. Padrão espacial do segundo fator comum da precipitação (escores), no Estado do Maranhão para o período de 1985 a 2014.

No mês de maio a região norte do Maranhão ainda sofre a influência da ZCIT, devido a sua proximidade com o Equador. Na faixa equatorial, o aquecimento da radiação solar é bastante uniforme e intenso, o que provoca baixas pressões à superfície, fazendo com que os ventos alísios de sudeste e nordeste convirjam em baixos níveis (Cavalcanti, 2012). Nos meses de junho, julho e agosto não ocorrem precipitação associada à ZCIT (que é o principal sistema indutor de precipitação na região) $\operatorname{logo}$, as contribuições do segundo fator nesses meses se devem as Linhas de Instabilidade (LI), que consistem em uma banda de nebulosidade de convecção profunda, que pode ser contínua ou não e atuam nestes meses e aos Distúrbios Ondulatórios de Leste - DOL, que são os principais sistemas produtores de precipitação no mês de julho na região. As LI e DOL correspondem, aproximadamente, de $25 \%$ a $30 \%$ do total de precipitação anual no período no setor noroeste do Maranhão. Os resultados corroboram com os encontrados por Barros e Oyama (2010), em estudo realizado em Alcântara no Norte do Maranhão.

Finalmente, o terceiro fator comum que explica $24,3 \%$ da variância total dos dados, apresenta correlações superiores a 0,6 nos meses de janeiro a abril (Figura 2). A configuração espacial do terceiro fator tem contribuições positivas em quase toda área ao norte, com exceção de uma pequena área no extremo nordeste e outro no centro noroeste do estado (Figura 5). Contribuições positivas com valores acima de 0,5 são visualizadas na parte sul do Maranhão no limite com o estado do Tocantins. Os sistemas de precipitação mais importantes atuantes nesse período são os VCAN nos meses de janeiro e fevereiro e a ZCIT em março e abril. Na região costeira as chuvas estão relacionadas ao deslocamento norte-sul da ZCIT sobre o Atlântico.

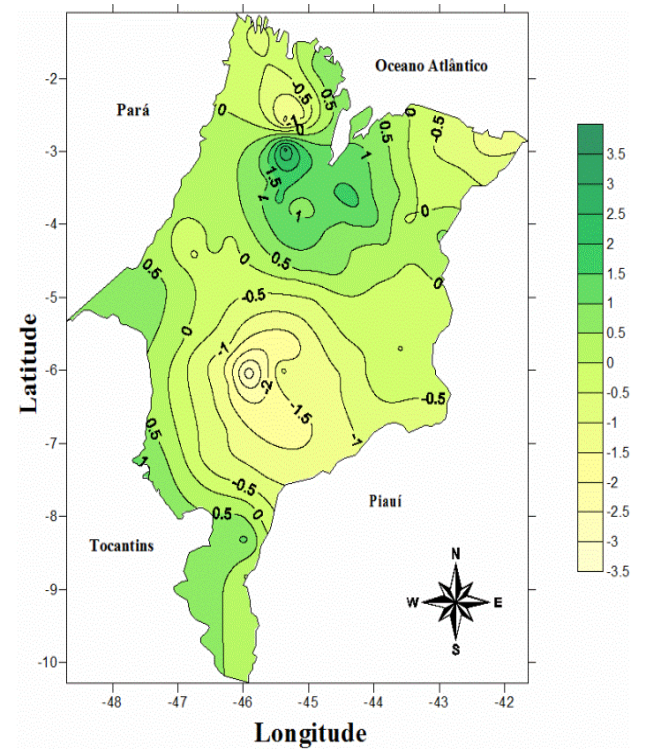

Figura 5. Padrão espacial do terceiro fator comum da precipitação (escores), no Estado do Maranhão para o período de 1985 a 2014. Regiões Homogêneas (RH) da Precipitação.

A determinação das cinco subregiões homogêneas da precipitação foi feita a partir dos fatores comuns temporais e espaciais mais signficativos que explicaram $92,8 \%$ da variância total dos dados. Para tanto, utilizou-se o método de agrupamento proposto por Ward (1963), por ser mais realístico com o regime pluviometrico do Estado (Figura 6). A seguir são apresentadas a descrição e características de cada uma das cinco regiões homogêneas.

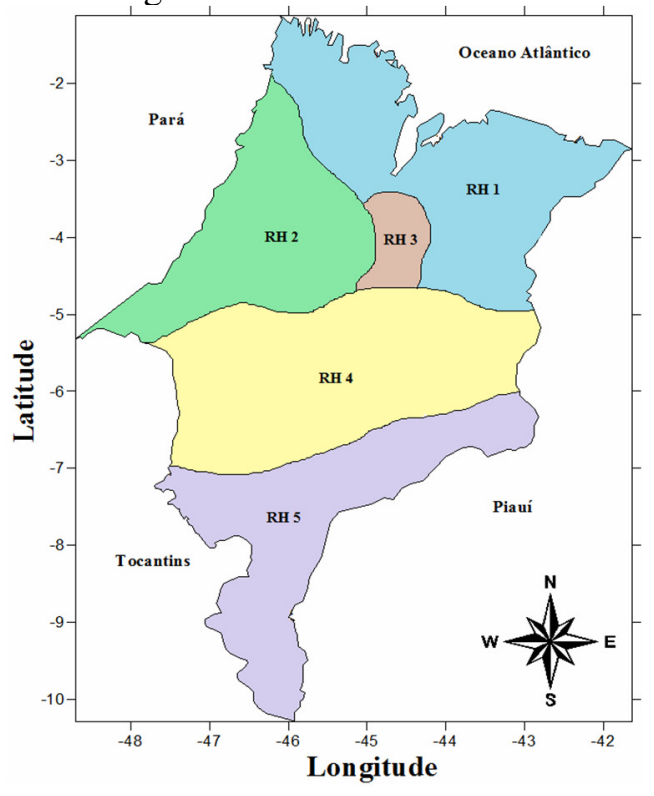

Figura 6. Regiões homogêneas da precipitação mensal para estado do Maranhão usando o método aglomerativo de Ward. 
A região homogênea RH1 situada na parte norte e nordeste do Estado é composta por 28 postos com precipitação média anual de $1955 \mathrm{~mm}$. A Figura 7 mostra o ciclo médio mensal da precipitação e seus respectivos desvios padrões. Observa-se que a precipitação média mensal máxima de $359,2 \mathrm{~mm}$ com desvio padrão de 76,6 mm ocorre em março e a mínima de 12,3 mm com desvio padrão de 9,9 $\mathrm{mm}$ em setembro. Constata-se que os maiores índices pluviométricos ocorrem de janeiro a maio, provavelmente devido à atuação dos VCAN e da ZCIT, respectivamente.

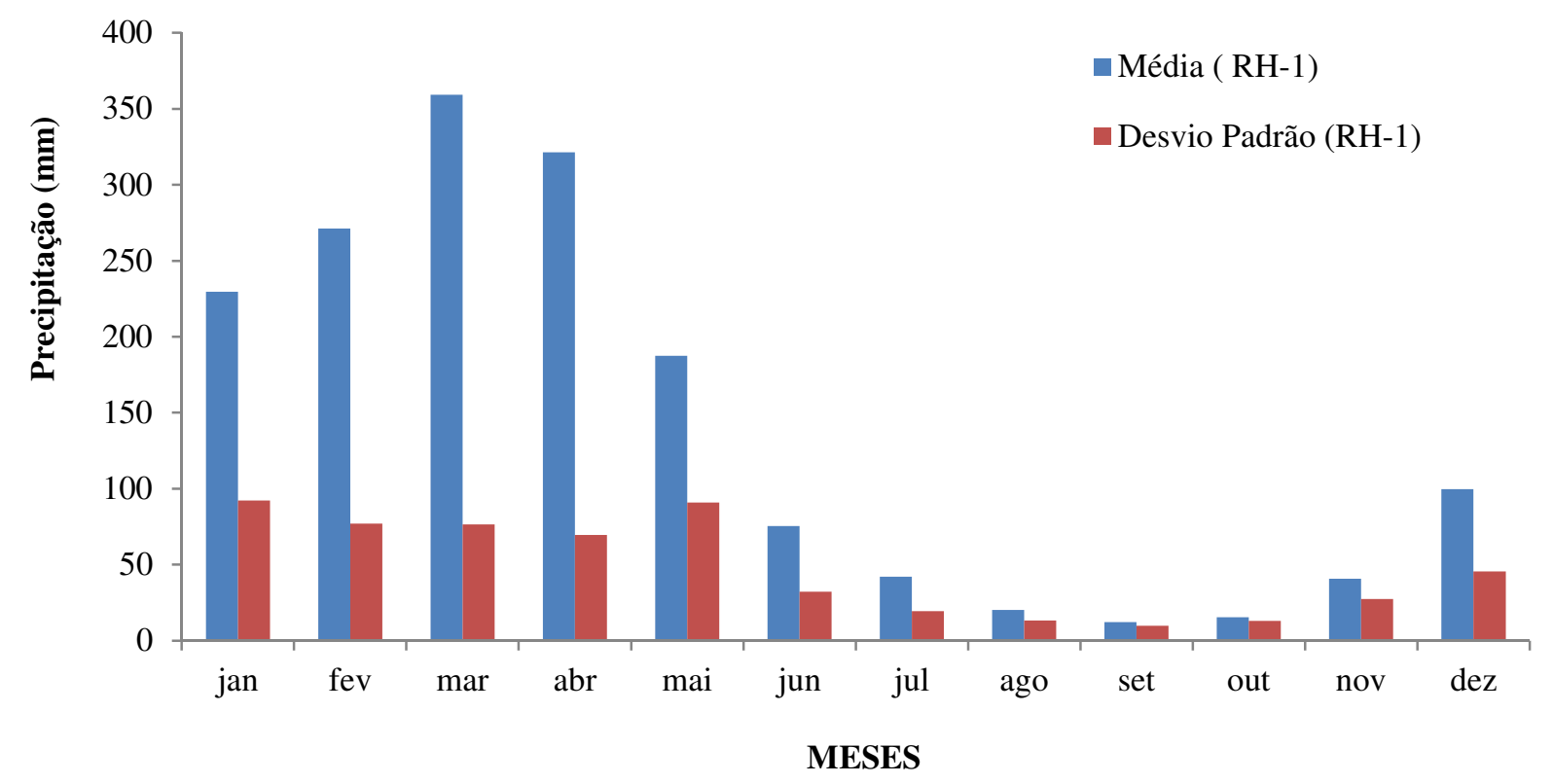

Figura 7. Valores médios mensais das precipitações e seus respectivos desvios padrões no período de 19852014 para os postos da RH1.

A região homogênea $\mathrm{RH} 2$ possui 13 postos, estende-se desde a latitude de $-2^{\circ}$ a $-5,5^{\circ} \mathrm{S}$, com precipitação média anual da ordem de $1597,8 \mathrm{~mm}$, em algumas localidades dessa $\mathrm{RH}$ os totais médios anuais superam os $1800 \mathrm{~mm}$. A máxima precipitação ocorre em março e a mínima em setembro. A maior concentração de chuvas ocorre nos primeiros meses do ano oriundos da atuação também dos VCAN, em janeiro e fevereiro, e da ZCIT, em março e abril. Apresenta comportamento médio semelhante ao da RH1, no entanto, no mês de dezembro a RH2 tem pluviosidade média superior a RH1 (Figura 8).

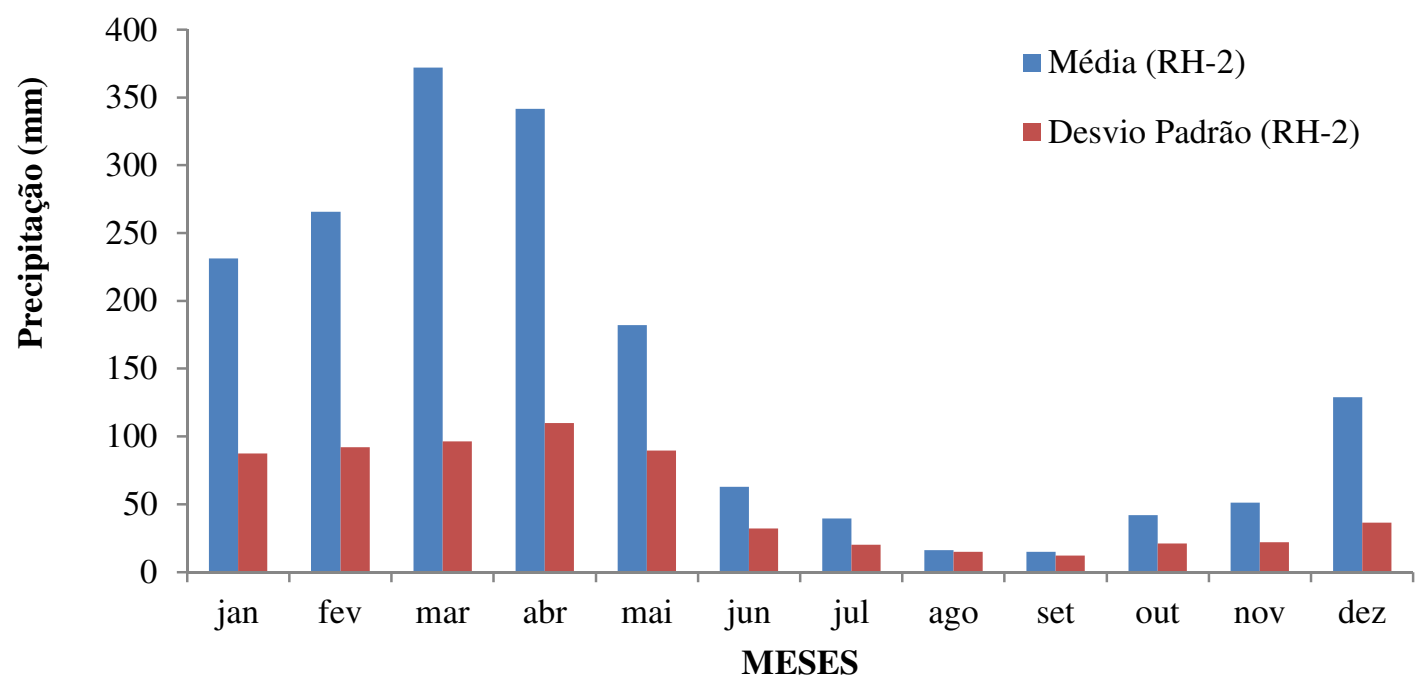

Figura 8. Valores médios mensais das precipitações e seus respectivos desvios padrões no período de 19852014, para os postos da RH2. 
A RH3 se encontra na parte central-norte do Estado constituída por 11 postos, com total médio anual de 1173,3 mm. A Figura 9 mostra o comportamento médio mensal da precipitação e seus respectivos desvios padrões. O período com maiores índices pluviométricos começa em dezembro e estende-se até maio, sendo março o mês mais chuvoso com 298,7 mm. Estes valores de precipitação pluvial são oriundos da atuação dos VCAN e ZCIT na região.

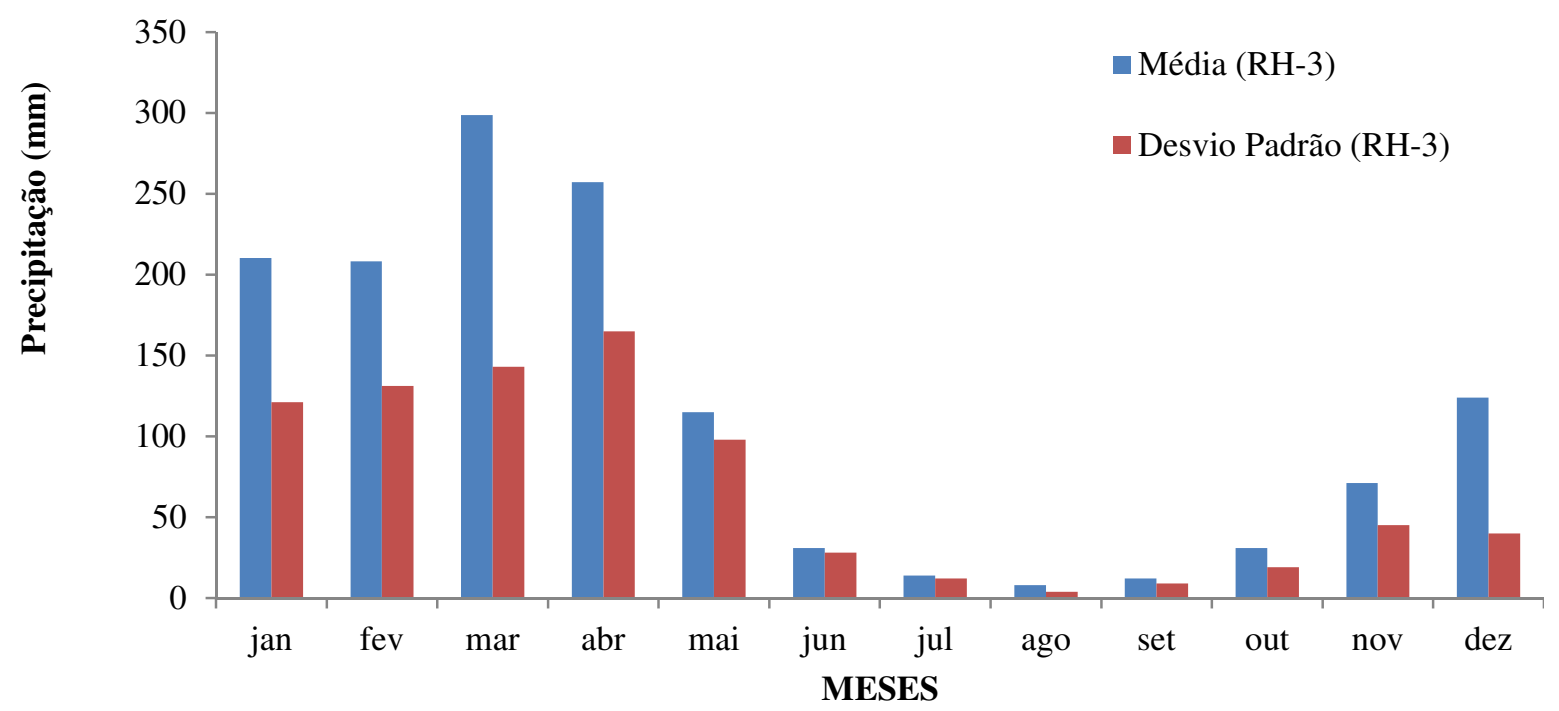

Figura 9. Valores médios mensais das precipitações e seus respectivos desvios padrões no período de 19852014, para os postos da RH3.

A quarta região RH4 composta por 18 postos pluviométricos localiza-se na parte central-sul do estado. Apresenta total médio anual de $1190 \mathrm{~mm}$. Pode-se observar na Figura 10 que o máximo de precipitação ocorre em março e mínimo em agosto. Nesta região o regime pluviométrico é influenciado pela atuação da ZCAS, Frentes, VCAN e ZCIT.

A região RH5 é formada por 15 postos e está localizada na parte Sul do estado. Essa região nos meses de novembro e dezembro sofre a influência da ZCAS e Sistemas Frontais que justificam as chuvas no período; em janeiro e fevereiro a precipitação na região está associada aos VCAN. Os maiores índices pluviométricos médios mensais são registrados de novembro a abril conforme Figura 11.

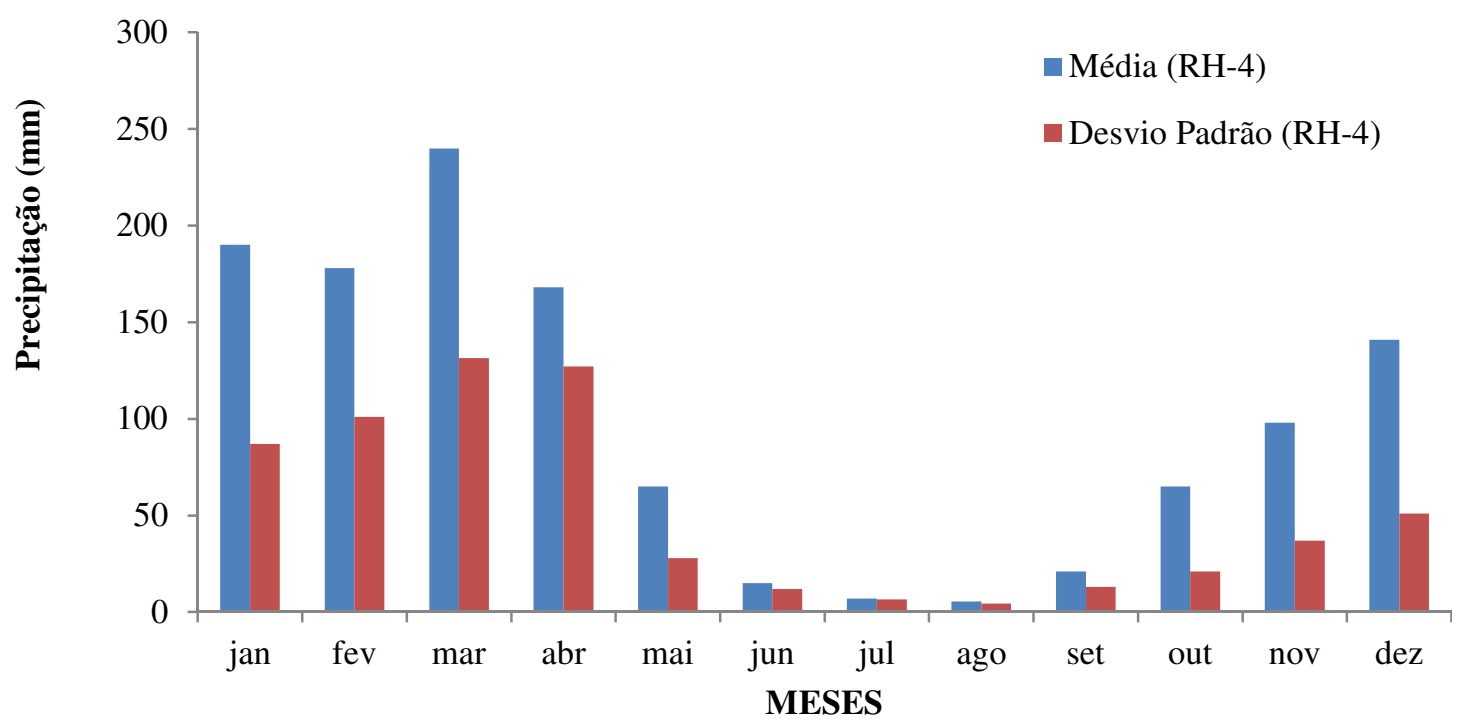

Figura 10. Valores médios mensais das precipitações e seus respectivos desvios padrões no período de 19852014, para os postos da RH4. 


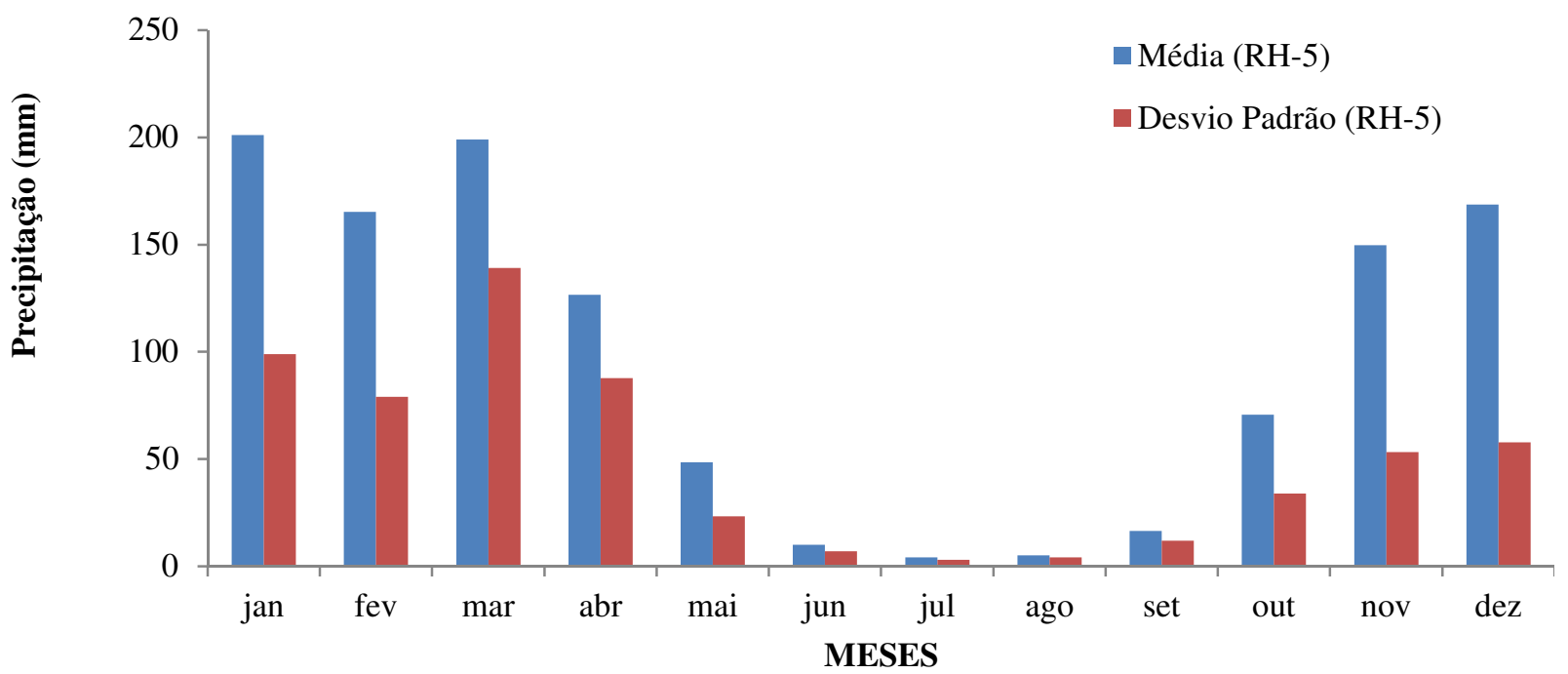

Figura 11. Valores médios mensais das precipitações e seus respectivos desvios padrões no período de 19852014, para os postos da RH5.

\section{Conclusões}

A análise fatorial em componentes principais (ACP) aplicada aos dados de chuva no Estado do Maranhão mostraram as regiões e períodos com altos e baixos índices pluviométricos, bem como os sistemas atmosféricos que influenciam na variabilidade espaço-temporal da precipitação na região. Foram definidos três padrões para o regime de chuvas no estado. O primeiro fator mostrou que no extremo sul as chuvas ocorrem em maior quantidade entre os meses de setembro a dezembro, influenciadas principalmente pela Zona de Convergência do Atlântico Sul e Sistemas Frontais, enquanto que nas regiões central e nordeste os valores são extremamente baixos. $\mathrm{O}$ segundo padrão, apresentou correlações bastante expressivas com as chuvas de maio a agosto, e está possivelmente relacionado a sistemas de grande e meso-escala, tal como a ZCIT, as Linhas de Instabilidade e os Distúrbios Ondulatórios de Leste, respectivamente. $\mathrm{O}$ terceiro padrão evidenciou chuvas mais intensas nos meses de janeiro a abril na parte norte do estado e no extremo com Tocantins, as quais são induzidas pela ZCIT, VCAS e influência Amazônica.

\section{Referências}

Amanajás, J.C., Braga, C.C., 2012. Padrões espaço-temporal Pluviométricos na Amazônia Oriental utilizando Análise Multivariada. Revista Brasileira de Meteorologia 27, 323-338.

Barros, S.S., Oyama, M.D., 2010. Sistemas Meteorológicos Associados à Ocorrência de Precipitação no Centro de Lançamento de Alcântara. Revista Brasileira de Meteorologia 25, 333-344.
Braga, C.C., 2000. Inter-relação entre padrões de índice de vegetação e de pluviometria no Nordeste do Brasil. Tese (Doutorado em Recursos Naturais). Universidade Federal da Paraíba.

Cavalcanti, I.F.A., 2012. Large scale and synoptic features associated with extreme precipitation over South America: A review and case studies for the first decade of the $21^{\text {st }}$ century. Atmospheric Research 118, 27-40.

Everitt, B., 1993. Cluster Analysis. Heinemann Educational Books, $3^{\mathrm{a}}$ ed. London.

Grimm, A.M., 2011. Interannual climate variability in South America: impacts on seasonal precipitation, extreme events, and possible effects of climate change. Stochastic Environmental Research and Risk Assessment 25, 537-554.

Jones, M., Blenkinsop, S., Fowler, H.J., Kilsby, C.G., 2014. Objective classification of extreme rainfall regions for the $\mathrm{UK}$ and updated estimates of trends in regional extreme rainfall. International Journal of Climatology 34, 751765.

Lorenz, E.N., 1956. Empirical orthogonal functions and statistical weather prediction. Sci. Rep. ${ }^{\circ}$ 1, Cambridge e Massachusetts (EUA), Statistical Forecasting Project Department of Meteorology, MIT.

Santos, S.A., Correia, M.F., Braga, C.C., Silva Aragão, M.R., 2010. Análise Estatística de Chuvas Intensas e Inundações no semiárido da bacia do rio São Francisco: a cheia de 1985. Engenharia Ambiental 7, 23.

Stathis, D., Myronidis, D., 2009. Principal component analysis of precipitation in Thessaly 
Revista Brasileira de Geografia Física V. 08 N. 02 (2015) 422-430.

Region (Central Greece). Global NEST Journal $11,467-476$.
Ward, J.H., 1963. Hierarchical grouping of optimized objective function. Journal of American Statistical Association 58, 236-244. 